\title{
Metabolic abnormalities in adult HIV infected population on antiretroviral medication in Malaysia: a cross-sectional survey
}

\author{
Nazisa Hejazi ${ }^{1}$, Roslee Rajikan ${ }^{1 *}$, Christopher Lee Kwok Choong ${ }^{2}$ and Suzana Sahar ${ }^{1}$
}

\begin{abstract}
Background: In the current two decades, dyslipidemia and increased blood glucose as metabolic abnormalities are the most common health threats with a high incidence among HIV/AIDS patients on antiretroviral (ARV) treatment. Scientific investigations and reports on lipid and glucose disorders among HIV infected communities are inadequate especially in those developing such as Malaysia. This cross-sectional survey was mainly aimed to evaluate the prevalence of metabolic abnormalities and associated risk factors among HIV infected population patients on ARV medication.
\end{abstract}

Methods: In a single reference health center in Malaysia, 2739 adult HIV positive patients on antiretroviral therapy (ART) were studied cross-sectionally using medical records. Besides demographic variables and associated health disorders, those factors which can change the lipid and glucose levels were collected. Logistic Regression was used to find the potential risk factors $(p<0.05)$.

Results: Majority of the studied population were male (81.1\%) and aged between 30-49 (68.6\%). Mean CD4 count was $474.25\left(\mathrm{cell} / \mathrm{s} / \mathrm{mm}^{3}\right.$ ) while undetectable RNA viral load was common among 83.3 (\%) of subjects. Among 1,583 patients with the recent blood lipid and glucose tests, increased levels of triglyceride (TG) and total cholesterol (TC) were frequently prevalent in half of the population as 59 (\%) and 54.2 (\%) while 28.7 (\%), 35.1 (\%) and 38.2 (\%) had declined level of high-density lipoprotein (HDL), raised low-density lipoprotein (LDL) and fasting plasma glucose (FPG) which were less common. Dyslipidemia was common in 82.3 (\%) of the subjects. Notably, medication with protease inhibitor (PI) was a potential risk for elevated triglyceride (odds ratio $(\mathrm{OR})=2.309,95 \%$ confidence interval $(C l)=1.605-3.324, P=0.001)$, high $T C(O R=1.561,95 \% C l=1.123-2.169, P=0.008)$ and low HDL $(\mathrm{OR}=1.449$, $95 \% \mathrm{Cl}=1.037-2.024, \mathrm{P}=0.029$ ). As lifestyle factor, alcohol consumption results as significant risk factor for raised TG $(\mathrm{OR}=2.653,95 \% \mathrm{Cl}=1.353-5.202, \mathrm{P}=0.004)$. Also having hepatitis raised risk of high $F P G$ level $(\mathrm{OR}=1.630$, $95 \% \mathrm{Cl}=1.197-2.220, \mathrm{P}=0.002$ ) in this sample population.

Conclusions: Dyslipidemia is highly common in Malaysian HIV subjects receiving ARV medication. Lifestyle modification, changing PI and switch to other ARV regimen can help in reduction of these abnormalities. Also suitable strategies and plans are necessary to prevent cardiovascular diseases in future.

Keywords: Lipid disorders, Metabolic abnormalities, Fasting plasma glucose, ARV medication, Protease inhibitor, Cardiovascular disease, Dyslipidemia, HIV positive, Malaysia

\footnotetext{
* Correspondence: Roslee.rr@gmail.com

'Dietetics Program, School of Health Sciences, Faculty of Health Sciences, Universiti Kebangsaan Malaysia, Jalan Raja Muda Abdul Aziz 50300, Kuala Lumpur, Malaysia

Full list of author information is available at the end of the article
} 


\section{Background}

Human immunodeficiency virus infection/acquired immunodeficiency syndrome (HIV/AIDS) is still considered as a tragic infectious disease with a high impact on quality of life among individuals and communities through the weakening economic and social performances which manifests along with poor mental and/or physical health. Based on the latest report by The Joint United Nations Programme on HIV/AIDS (UNAIDS), of 34.0 million [31.4 million- 35.9 million] people living with HIV (PLHIV) at the end 2011 universally [1]. Roughly 81,000 of them [2] were resident in Malaysia which is a south-east Asian and Upper middle-income country [3]. Indeed the number of PLHIVs receiving ART as of December 2011 has increased up to 14,002 [2]. In combat with HIV/AIDS, there are some progresses in heath condition, quality of life and life expectancy specific after introduction of antiretroviral therapy (ART) as the only available medication for control of this disease [4]. However there has been discovered some adverse effects of ART in the form of glucose and/or lipid abnormalities which are accounted as cardiovascular risk factors [5]. The prevalence rates for these disorders are widely varied ranging from less than $30 \%$ for elevated glucose level [6-9] and 10\%-20\% for diabetes [10-13], $10-60 \%$ for hypercholesterolemia [14-17], 20-70\% for hypertriglyceridemia [15,18,19], 20-40\% for low HDL level [18-20], which are mostly in the form of elevated triglyceride and low HDL level. In reviews by Carr et al. (1998), Carr (2000) and Currier et al. (2008) on substantial HIV/AIDS studies they explained other than traditional risk factors and HIV infection itself [21-23]. They stressed that protease inhibitor (PI) as the third antiretroviral (ARV) class also is responsible for these complications in comparison with other ARV categories such as nucleoside analog reverse transcriptase inhibitors (NRTI) and non-nucleoside analog reverse transcriptase inhibitors (NNRTI) and integrase inhibitors (INI).

Generally, the knowledge on blood metabolic abnormalities among PLHIV have established mostly in developed societies including USA [24-26], Europe [27,28], Australia [29-32] and limited information in developing [16,19,33,34] and African countries [35-37].

In Asian and specific South east Asian countries estimated incidence of lipid abnormalities in HIV/AIDS mostly are reported from Thailand [15,33,38-40] and India $[7,41,42]$. They found a very wide range of $5-60 \%$ for high TC, high LDL-c, high TG and low HDL among antiretroviral therapy (ART) individuals. Elevated FPG also is estimated between estimated $27 \%$ among Thai $[6,38]$ and $60 \%$ in Indian HIV population [7].

The aim of this study is to provide some evidences on metabolic abnormalities and also associated risk factors since there is very limited information from HIV/AIDS studies about these disorders in Malaysia. Moreover, information from the developed countries cannot be deduced to developing societies due to differences in patient populations, HIV subtypes and lifestyle therefore, therefore initial and additional studies from developing countries are required. we assessed the prevalence of lipid and glucose abnormalities and also associated the risk factors with each these abnormalities among Malaysian HIV-infected patients in this study.

\section{Methods}

\section{Study population and design}

This cross-sectional study was conducted between January to September 2012 at an Infectious Diseases (ID) clinic which is also a reference center for HIV and AIDS cases, located in Selangor, Malaysia. All HIV patients who started their ARV medication till first January 2012 were listed based on the computerized medical records. Patients included in this study met the following criteria: subjects must have been on antiretroviral medication at least for 6 months and stable HAART for at least 4 weeks before study entry, had complete or at least partial lipid and glucose panel performed during this study, equal or more than 20 years of age and hold Malaysian citizenship. All death cases were excluded.

Ethical approval was obtained from research ethic committee/IRB National University of Malaysia and National Medical Research Registry (NMRR) Malaysia.

\section{Methods}

The collected secondary data from computerized medical records comprised of demographics (age, gender, and ethnicity), weight, height and blood pressure, multivitamin supplementation, ARV medication (NRTIS, NNRTIS, PIs), those medication history which have effect on lipid and or glucose level (anti-hypertensive, diuretic, steroid/hormone, lipid-lowering, anti-hyperglycemic). Also information regarding severe opportunistic infections during preceding six months, medical history as the major current health problem (tuberculosis, diabetes mellitus, hepatitis, other liver and renal diseases), alcohol and smoking status including the number of subject as current alcohol consumer, current smoker, and the number of smoked cigarettes per day were collected.

Fasting blood samples were collected for all studied subjects. Laboratory testing for blood samples included the following: fasting lipid profile, fasting plasma glucose, CD4 count, RNA viral load. The laboratory methods for determination of lipid profiles includes Cholesterol OxidasePeroxidase Amino Phenazone Phenol (CHOD-PAP) for TC, Accelerator Selective Detergent for HDL,Glycerol-3Phosphate Oxidase-Peroxidase Amino Phenazone Phenol (GPO-PAP) for TG while Low-density lipoprotein (LDL) was derived from an indirect measurement using the 
Friedewald formula [43] . Blood TC, TG and HDL cholesterol was tested using the Cobas Integra 400 Plus analyzer (Roche Diagnostics Ltd. CH6343, Rotkreuz, Switzerland). Blood glucose was tested by CD4 was measured using the FACS Calibur system (Becton Dickinson, San Jose, CA). HIV RNA Viral load was detected with Immunophenotyping method using $\mathrm{COBAS}^{\circ} \mathrm{AMPLICOR}$ analyser.

\section{Study definitions}

Lipid abnormalities were defined according tothe National Cholesterol Education Program (NCEP) Adult Treatment Panel III (ATP III) criteria in 2001 [44] while the American Heart Association (AHA) and the National Heart, Lung, and Blood Institute (NHLBI)/Updated NCEP $[45,46]$ criteria was applied for plasma glucose level. Abnormalities in lipid profile and glucose level $(\mathrm{mmol} / \mathrm{l})$ were classed as $\mathrm{TG} \geq 1.7, \mathrm{LDL} \geq 3.36, \mathrm{TC} \geq 5.17, \mathrm{HDL}<1.03$ (male) and $\mathrm{HDL}<1.30$ (female), FPG $\geq 5.6$. The definition of dyslipidemia was according to the occurrence of one or more of the lipid abnormalities (TG, TC, LDL, HDL) according to the criteria of the National Cholesterol Education Program Adult Treatment Panel III.

The CD4 cell counts (cells $/ \mathrm{mm}^{3}$ ) were categorized in there groups $(<200,200-499, \geq 500)$ according to the standard of Centre for Disease Control and Prevention [47]. Also HIV RNA load (copies/mL) was classified using the recommendation from International AIDS Society-USA Expert Panel [48] references into undetectable level (value below 20) and detectable level (as equal or greater than 20).

\section{Statistical}

Analysis

All collected data were analyzed using the statistical packages for social science (SPSS) for window, version 16.0 [49]. Descriptive data in the form of mean \pm standard deviation (SD) and frequency as the number and percent (\%) were tested. At the beginning, all variables were tested for normality distribution and then data cleaning was carried out to weed out the out-of-range numbers. At the second step of data analysis, based on the lipid and glucose data (normal and non-normal groups) the means and percentage distribution of each variable were calculated using independent sample-t-test and chi-square test of homogeneity to find significant differences and associations in variables. As the last step, the significant risk factors for each metabolic abnormality using adjusted odd ratios, 95\% confidence intervals and p-values were identified through univariate test as binary logistic regression with enter method. All binary analyses were adjusted for age, gender, ethnicity, hypertension, diabetes mellitus, CD4, HIV RNA Load, TC, TG, LDL, HDL, FPG, alcohol consumption, smoking, liver disease, medication with agents including ARV (PI), b-blocker, anti-hyperglycemic. The significance tests were 2-sided, and P values less than 0.05 were considered statistically significant.

\section{Results}

\section{Characteristics of study population}

Two thousand seven hundred thirty nine patients were included in the analysis. Dyslipidemia was common in $82.3 \%$ of 1583 HIV subjects. Demographics are shown in Table 1. Most subjects were aged between 40 to 59 years (55.6\%), Chinese $(58.8 \%)$ and male $(81.1 \%)$. Mean systolic $(121.31 \pm 16.84 \mathrm{mmHg})$ and diastolic blood pressure $(76.83 \pm 11.40 \mathrm{mmHg})$ were in normal range while $19.7 \%$ of 2670 subjects were hypertensive. In terms of ARV medication of all 2637 subjects on ARV treatment 9.3\% was receiving PIs and the rest were on the combination of NRTIs + NNRTIs medication. Small proportions of subjects were taking anti-hypertension agents (12.2\%) and lipid lowering (18.5\%). Majority of HIV study population $(89.2 \%)$ had CD4 cell count $\geq 200$ (cells $/ \mathrm{mm}^{3}$ ) and also $83.3 \%$ had a well suppressed and undetectable HIV viral load as less than 20 (copies $/ \mathrm{mm}^{3}$ ). In relation to lipid abnormalities, a smaller group of subjects (26.7\%) had the lipid test result only for year 2011 in the time of data collection. The lipid profile results from year 2012 showed that increased level of triglyceride $(>1.7 \mathrm{~mol} / \mathrm{ml})$ was common among $59.1 \%$ of subjects while $35.1 \%$ and $54.2 \%$ of subjects had higher amount of LDL and total cholesterol. HDL was lower than normal level in $28.7 \%$ of subjects and $38.2 \%$ had high fasting plasma glucose level.

Also in year 2012 till September $10.5 \%$ of study population were current smoker that $46.5 \%$ of smokers reported more than 20 smoked cigarettes per day. Prevalence of diabetes mellitus and Hepatitis are estimated as 12.9\% and $14.4 \%$ as the most common medical associated condition among this population. HDL (Mean $+\mathrm{SD}$ ) level in male $(0.89+0.12 \mathrm{mmol} / \mathrm{l})$ was lower than and female $(1.08+$ $0.17 \mathrm{mmol} / \mathrm{l})$ groups.

\section{Risk factors of high TG}

The result of logistic regression analysis (Table 2) revealed that significant risk factors $(p<0.001)$ for increased TG level were increasing age $(\mathrm{OR}=1.018,95 \%$ $\mathrm{CI}=1.008-1.029)$, having hypertension $(\mathrm{OR}=1.516,95 \%$ $\mathrm{CI}=1.173-1.960)$ and diabetes mellitus $(\mathrm{OR}=1.532$, $95 \% \mathrm{CI}=1.150-2.040)$, taking b-blockers as antihypertensive agents $(\mathrm{OR}=1.668,95 \% \mathrm{CI}=-1.042)$, higher FPG $(\mathrm{OR}=1.166,95 \% \mathrm{CI}=1.084-1.253)$, higher CD4 cell count $(\mathrm{OR}=1.001,95 \% \mathrm{CI}=1.001-1.002)$, higher level of $\mathrm{TC}(\mathrm{OR}=1.281,95 \% \mathrm{CI}=1.179$ - 1.392) with following strong risks as low $\mathrm{HDL}$ level $(\mathrm{OR}=3.585$, $95 \% \mathrm{CI}=2.779-4.625)$, alcohol taking $(\mathrm{OR}=2.653$, 95\% 
Table 1 Characteristics of 2739 HIV infected Subjects on ARV medications

\begin{tabular}{lcc}
\hline Characteristics & N (\%) & Mean \pm SD \\
\hline Age & & $43.06 \pm 9.90$ \\
$20-39$ & $1053(38.5)$ & $33.56 \pm 4.35$ \\
$40-59$ & $1524(55.6)$ & $47.22 \pm 5.14$ \\
$\geq 60$ & $162(5.9)$ & $65.70 \pm 4.82$
\end{tabular}

\section{Ethnicity}

Malay

$836(30.5)$

Chinese

$1611(58.8)$

Indian

Other '

$274(10.0)$

$18(0.7)$

Gender

Male

$2220(81.1)$

Female

Weight (Kg)

Height (cm)

Hypertension*

$519(18.9)$

$2582(94.27)$

$215(7.85)$

$61.85 \pm 1.21$

$514(19.7)$

ARV without PI exposure

2392 (90.7)

ARV with PI exposure

$245(9.3)$

384 (14)

70 (2.6)

$263(9.6)$

$15(0.5)$

$6(0.2)$

$508(18.5)$

$191(7.0)$

$2739(100.0)$

$474.25 \pm 263.02$

292 (10.8)

$122.94 \pm 53.78$

2282 (83.3)

933(59.1)

$3.27 \pm 1.92$

$555(35.1)$

$4.10 \pm 0.75$

857 (54.2)

$6.16 \pm 0.95$

454 (28.7)

$588(38.2)$

$7.08 \pm 2.24$

1303 (82.3)

$83(3.0)$

$288(10.5)$

$352(12.9)$

$46(1.7)$

$395(14.4)$

$51(1.9)$

$\mathrm{CI}=1.353-5.202)$ and ARV therapy with PIs $(\mathrm{OR}=2.309$, $95 \% \mathrm{CI}=1.605-3.324)$.

On the other hand being female $(\mathrm{OR}=0.550,95 \%$ $\mathrm{CI}=0.428-0.707)$ and Malay $(\mathrm{OR}=0.676,95 \% \mathrm{CI}=$ 0.464- 0.985), not taking anti- hyperglycemic agents $(\mathrm{OR}=0.603,95 \% \mathrm{CI}=0.419-0.870)$, higher HDL level $(\mathrm{OR}=0.485,95 \% \mathrm{CI}=0.384-0.612)$, having hepatitis disease $(\mathrm{OR}=0.576,95 \% \mathrm{CI}=0.424-0.781)$ significantly reduce the risk of hypertriglyceridemia $(\mathrm{p}<0.001)$. CD4 cell, viral load, LDL level and smoking were not associated with high TG level significantly $(\mathrm{p}>0.05)$.

\section{Risk factors of increased LDL}

In this study age, gender, taking ARV agents, medication with anti-hyperglycemic drugs, diabetes, smoking, alcohol consumption, CD4 cell, viral load and FPG level were not significant risk factor for high LDL ( $p>0.05)$. Having hypertension $(\mathrm{OR}=1.405,95 \% \mathrm{CI}=1.093-1.805)$ and diabetes mellitus $(\mathrm{OR}=1.532,95 \% \mathrm{CI}=1.150-2.040)$, higher TC level $(\mathrm{OR}=6.468,95 \% \mathrm{CI}=5.319-7.866)$ with following strong risk as normal HDL level $(\mathrm{OR}=2.331$, $95 \% \mathrm{CI}=1.812-2.997)$ increased the risk $(\mathrm{p}<0.05)$ for high LDL level (Table 3).

Conversely being Chinese $(\mathrm{OR}=0.551,95 \% \mathrm{CI}=0.434-$ $0.701)$, lower TG level $(\mathrm{OR}=0.858,95 \% \mathrm{CI}=0.800-0.920)$, and having hepatitis disease $(\mathrm{OR}=0.592,95 \% \mathrm{CI}=0.419$ $0.836)$, significantly protect the subjects from increased LDL level $(\mathrm{p}<0.001)$.

\section{Risk factors of increased TC}

Also the findings of this study (Table 4) confirmed that that significant risk factors $(\mathrm{p}<0.001)$ for elevated TC were being older ( $\mathrm{OR}=1.01095 \% \mathrm{CI}=1.000-1.021)$, being female $(\mathrm{OR}=1.500,95 \% \mathrm{CI}=1.163-1.936)$, having hypertension $(\mathrm{OR}=1.440,95 \% \mathrm{CI}=1.122-1.848)$, having viral load level $<20$ copies $/ \mathrm{mm}^{3}(\mathrm{OR}=1.460$, 95\% $\mathrm{CI}=1.093-1.951)$, higher level of LDL (OR = 6.977, 95\% CI =5.705-8.534), higher TG level $(\mathrm{OR}=$ $1.312,95 \% \mathrm{CI}=1.220-1.412$ ), having normal HDL level $(\mathrm{OR}=1.867,95 \% \mathrm{CI}=1.498-2.327)$ and treatment with PI $(\mathrm{OR}=1.561,95 \% \mathrm{CI}=1.123-2.169)$.

Interesting that having hepatitis disease $(\mathrm{OR}=0.527$, $95 \% \mathrm{CI}=0.387-0.718)$ significantly protect the subjects from elevated total cholesterol $(\mathrm{p}<0.001)$. Other factors including ethnicity, b-blocker, anti-hyperglycaemic medications, diabetes mellitus, FPG, CD4, smoking and alcohol consumption did not result in high level of TC significantly $(\mathrm{p}>0.05)$.

\section{Risk factors of low HDL}

Also Table 5 shows that the risk of low HDL level was significantly ( $\mathrm{p}<0.001$ ) related to the factors including being Chinese $(\mathrm{OR}=1.753,95 \% \mathrm{CI}=1.187-2.589)$, taking antihyperglycemic agent $(\mathrm{OR}=1.636,95 \% \mathrm{CI}=1.152-2.323)$, 
Table 2 Risk factors for increased triglyceride (TG) in 1579 HIV subjects (normal =646, increased TG =933) on ARV medication

\begin{tabular}{|c|c|c|c|c|c|c|}
\hline \multirow[t]{2}{*}{ Characteristics } & \multirow{2}{*}{$\begin{array}{c}\text { Normal } \\
\text { Mean } \pm \text { S.D. } \\
\text { N (\%) }\end{array}$} & \multirow{2}{*}{$\begin{array}{c}\text { Increased triglyceride } \\
\text { Mean } \pm \text { S.D. } \\
\mathrm{N}(\%)\end{array}$} & \multirow[t]{2}{*}{$\mathrm{aOR}$} & \multicolumn{2}{|c|}{ 95.0 C.I. for OR } & \multirow[t]{2}{*}{$p$-value } \\
\hline & & & & Lower & Upper & \\
\hline Age (per year) & $43.41 \pm 10.51$ & $45.14 \pm 9.36$ & 1.018 & 1.008 & 1.029 & 0.001 \\
\hline \multicolumn{7}{|l|}{ Gender } \\
\hline Male & $484(74.9)$ & 788 ( 84.5) & 1.00 & & & \\
\hline Female & $162(25.1)$ & $145(15.5)$ & 0.550 & 0.428 & 0.707 & 0.001 \\
\hline
\end{tabular}

Ethnicity

$\begin{array}{lc}\text { Indian } & 62(9.7) \\ \text { Malay } & 190(29.8) \\ \text { Chinese } & 386(60.5)\end{array}$

Hypertension

No $515(82.4)$

Yes $\quad 110(17.6)$

ARV medication

\section{Without PI}

$591(93.6)$

With PI

$$
42 \text { (6.4) }
$$

$786(85.9)$

$129(14.1)$

872 (93.5)

Not taking

$620(96.0)$

Taking

$$
26 \text { (4.0) }
$$

$61(6.5)$

103 (11.0)

830 (89.0)

601 (93.0)

$565(77.5)$

$81(12.5)$

$5.05 \pm 1.00$

$363(56.2)$

$<5.17$

$283(59.1)$

$\geq 5.17$

$1.43 \pm 0.44$

HDL (mmol/l)

Normal $^{\S}$

551 (85.3)

Low

95 (14.7)

FPG (mmol/l)

$5.52 \pm 1.37$

Normal

High

432 (68.7)

197 (31.3)

Alcohol

Not Taking

635 (98.3)

Taking

$11(1.7)$

Hepatitis

No

100 (15.5)

Yes

89 (9.5)

$$
\begin{aligned}
& 765(82.0) \\
& 186(18.0) \\
& 5.43 \pm 1.40 \\
& 361(38.7) \\
& 571(40.9) \\
& 1.24 \pm 0.61 \\
& 576(61.8) \\
& 356(38.2) \\
& 5.91 \pm 1.95 \\
& 519(57.2) \\
& 389(42.8)
\end{aligned}
$$

892 (95.6)

41 (4.4)

844 (90.5)
1.668

1.042

2.670

0.603

0.419

0.870

0.006

1.00

1.532

1.150

2.040

0.004

1.281

1.179

1.392

0.001

1.00

2.029

1.655

2.487

0.001

0.485

0.384

0.612

0.001

1.00

3.585

2.779

4.625

0.001

1.166

1.084

1.253

0.001

1.00

1.644

1.327

2.035

0.001

1.00

2.653

1.353

5.202

0.004
0.424

0.781
${ }^{5} \geq 1.03$ ( Male) and $\geq 1.30$ (Female). ARV: Antiretroviral. Adjusted for age, gender, ethnicity, hypertension, diabetes mellitus, CD4, HIV RNA Load, TC, LDL, HDL, FPG, alcohol consumption, smoking, liver disease, medication with agents including ARV (PI), b-blocker, anti-hyperglycemic. aOR Adjusted odds ratio. 
Table 3 Risk factors for increased low-density lipoprotein cholesterol (LDL-C) level in 1578 HIV subjects (normal = 1023, increased LDL-C $=555$ ) on ARV medication

\begin{tabular}{|c|c|c|c|c|c|c|}
\hline \multirow[t]{2}{*}{ Characteristics } & \multirow{2}{*}{$\begin{array}{c}\text { Normal } \\
\text { Mean } \pm \text { S.D. } \\
\text { N (\%) }\end{array}$} & \multirow{2}{*}{$\begin{array}{c}\text { Increased LDL } \\
\text { Mean } \pm \text { S.D. } \\
\text { N (\%) }\end{array}$} & \multirow[t]{2}{*}{$\mathrm{aOR}$} & \multicolumn{2}{|c|}{ 95.0 C.I. for OR } & \multirow[t]{2}{*}{ p-value } \\
\hline & & & & Lower & Upper & \\
\hline \multicolumn{7}{|l|}{ Ethnicity } \\
\hline Malay & 703 (69.3) & 317 (57.4) & 1.00 & & & \\
\hline Chinese & $214(21.1)$ & 175 (31.7) & 0.551 & 0.434 & 0.701 & 0.001 \\
\hline Indian & $98(9.7)$ & $60(10.9)$ & 0.749 & 0.513 & 1.093 & 0.134 \\
\hline \multicolumn{7}{|l|}{ Hypertension } \\
\hline No & 785 (80.5) & 400 (74.6) & 1.00 & & & \\
\hline Yes & 190 (19.5) & $136(25.4)$ & 1.405 & 1.093 & 1.805 & 0.008 \\
\hline TG $(\mathrm{mmol} / \mathrm{l})$ & $2.54 \pm 2.00$ & $2.14 \pm 1.14$ & 0.858 & 0.800 & 0.920 & 0.001 \\
\hline$<3.36$ & $413(40.4)$ & $233(42.0)$ & 1.00 & & & \\
\hline$\geq 3.36$ & 609 (59.6) & $322(58.0)$ & 0.937 & 0.760 & 1.156 & 0.545 \\
\hline TC $(\mathrm{mmol} / \mathrm{l})$ & $4.74 \pm 1.00$ & $6.26 \pm 1.09$ & 6.468 & 5.319 & 7.866 & 0.001 \\
\hline$<5.17$ & 705 (69.0) & $19(3.4)$ & & & & \\
\hline$>5.17$ & $317(31.0)$ & 536 (96.6) & 2.620 & 2.399 & 2.861 & 0.001 \\
\hline HDL (mmol/l) & $1.31 \pm 0.65$ & $1.33 \pm 0.33$ & 1.049 & 0.876 & 1.257 & 0.602 \\
\hline Low & $349(34.1)$ & $101(18.2)$ & 1.00 & & & \\
\hline Normal ${ }^{\S}$ & $673(65.9)$ & 454 (81.8) & 2.331 & 1.813 & 2.997 & 0.001 \\
\hline \multicolumn{7}{|l|}{ Hepatitis } \\
\hline No & $882(86.2)$ & 507 (91.4) & 1.00 & & & \\
\hline Yes & 141 (13.8) & $48(8.6)$ & 0.592 & 0.419 & 0.836 & 0.003 \\
\hline
\end{tabular}

$\S \geq 1.03$ (Male) and $\geq 1.30$ (Female). Adjusted for age, gender, ethnicity, hypertension, diabetes mellitus, CD4, HIV RNA Load, TC, TG, HDL, FPG, alcohol consumption, smoking, liver disease, medication with agents including ARV (PI), b-blocker, anti-hyperglycemic. aOR Adjusted odds ratio.

higher LDL level $(\mathrm{OR}=1.709,95 \% \mathrm{CI}=1.516-1.926)$, increased level of $\mathrm{TC} \geq 5.17(\mathrm{OR}=1.867,95 \% \mathrm{CI}=1.498-$ 2.327) and ARV therapy with PIs (OR $=1.449,95 \% \mathrm{CI}=$ 1.037-2.024). Beside this study showed that being Indian $(\mathrm{OR}=0.570,95 \% \mathrm{CI}=0.386-0.842)$, higher level of $\mathrm{TG}$ $(\mathrm{OR}=0.693,95 \% \mathrm{CI}=646-0.745)$ and having hepatitis disease $(\mathrm{OR}=0.630,95 \% \mathrm{CI}=0.459-0.865)$ significantly decreased the risk of low HDL level $(\mathrm{p}<0.001)$. Age, gender, hypertension, b-blocker, diabetes mellitus, FPG, alcohol consumption, smoking were not associated with low level of HDL.

\section{Risk factors of raised FPG}

Table 6 present those significant factors $(\mathrm{p}<0.001)$ which increase the risk of hyperglycemia including increasing age $(\mathrm{OR}=1.048,95 \% \mathrm{CI}=1.036-1.059)$, having hypertension $(\mathrm{OR}=1.954,95 \% \mathrm{CI}=1.522-2.508)$ and higher level of TG (OR $=1.130,95 \% \mathrm{CI}=1.066-1.199)$.

On the other hand being female $(O R=0.711,95 \%$ $\mathrm{CI}=0.543$ - 0.931), not taking b-blocker $(\mathrm{OR}=0.585$, 95\% CI $=0.379$ - 0.902), not taking anti-hyperglycemic agents $(\mathrm{OR}=0.164,95 \% \mathrm{CI}=0.110-0.244)$ and not having diabetes mellitus $(\mathrm{OR}=0.330,95 \% \mathrm{CI}=0.249-0.438)$ significantly protect the subjects from increased fasting plasma glucose $(\mathrm{p}<0.001)$.

Differences in ethnicity, using PI, CD4 viral load level, LDL, TC, HDL, alcohol consumption and smoking were not increased the risk of high FPG significantly $(\mathrm{p}>0.05)$.

\section{Discussion}

This primarily and large study of HIV infected subject on ART found that dyslipidemia was a high predominant disorder. Majority of the subjects (60\%) had increased levels of TG or TC while a less proportions of subject affected with high levels of LDL (35.1\%) and FPG (38.2\%) and, low HDL level (28.7\%). Some studies in Thailand reported a similar rate of dyslipidemia at $53.6 \%$ and $88 \%$ [38,50]. Other studies from other developing countries showed similar results oh high prevalence lipid abnormalities rate of more than 76\% [35] from Tanzania, 82.3\% [19] from Southern Ethiopia, 20$100 \%$ from India $[7,41]$.

Disturbances in level of triglyceride and total cholesterol were more prevalent than HDL and LDL in this study. Generally increases in TG and TC level are mainly 
Table 4 Risk factors for increased total cholesterol (TC) level in 1582 HIV subjects (normal $=725$, increased TC $=857$ ) on ARV medication

\begin{tabular}{|c|c|c|c|c|c|c|}
\hline \multirow[t]{2}{*}{ Characteristics } & \multirow{2}{*}{$\begin{array}{c}\text { Normal } \\
\text { Mean } \pm \text { S.D. } \\
\text { N (\%) }\end{array}$} & \multirow{2}{*}{$\begin{array}{c}\text { Increased TC } \\
\text { Mean } \pm \text { S.D. } \\
\text { N (\%) }\end{array}$} & \multirow[t]{2}{*}{$\mathrm{aOR}$} & \multicolumn{2}{|c|}{ 95.0 C.I. for OR } & \multirow[t]{2}{*}{ p-value } \\
\hline & & & & Lower & Upper & \\
\hline \multicolumn{7}{|l|}{ Age (year) } \\
\hline Mean \pm SD & $43.87 \pm 10.09$ & $44.88 \pm 9.65$ & 1.010 & 1.000 & 1.021 & 0.043 \\
\hline \multicolumn{7}{|l|}{ Gender } \\
\hline Male & $608(83.9)$ & $665(77.6)$ & 1.00 & & & \\
\hline Female & $117(16.1)$ & $192(22.4)$ & 1.500 & 1.163 & 1.936 & 0.002 \\
\hline \multicolumn{7}{|l|}{ Hypertension } \\
\hline No & $565(81.6)$ & $621(75.5)$ & 1.00 & & & \\
\hline Yes & $127(18.4)$ & $201(24.5)$ & 1.440 & 1.122 & 1.848 & 0.004 \\
\hline \multicolumn{7}{|l|}{ ARV medication } \\
\hline Without PI & $649(91.3)$ & $731(87.0)$ & 1.00 & & & \\
\hline With PI & $62(8.7)$ & $109(13.0)$ & 1.561 & 1.123 & 2.169 & 0.008 \\
\hline \multicolumn{7}{|c|}{ Viral load (copies/mL) } \\
\hline$\geq 20$ & $115(15.9)$ & $98(11.4)$ & 1.00 & & & \\
\hline$<20$ & $610(84.1)$ & $759(88.6)$ & 1.460 & 1.093 & 1.951 & 0.010 \\
\hline LDL (mmol/l) & $2.37 \pm 0.72$ & $3.57 \pm 0.97$ & 6.977 & 5.705 & 8.534 & 0.001 \\
\hline$<3.36$ & $705(97.4)$ & $317(37.2)$ & 1.00 & & & \\
\hline$\geq 3.36$ & $19(2.6)$ & $536(62.8)$ & 20.150 & 12.928 & 31.406 & 0.001 \\
\hline TG $(\mathrm{mmol} / \mathrm{l})$ & $2.03 \pm 1.29$ & $2.73 \pm 2.09$ & 1.312 & 1.220 & 1.412 & 0.001 \\
\hline$<5.17$ & $363(50.1)$ & $283(33.1)$ & 1.00 & & & \\
\hline$\geq 5.17$ & 361 (49.9) & 571 (66.9) & 2.029 & 1.655 & 2.487 & 0.001 \\
\hline $\mathrm{HDL}(\mathrm{mmol} / \mathrm{l})$ & $1.31 \pm 0.70$ & $1.32 \pm 0.40$ & 1.034 & 0.866 & 1.234 & 0.714 \\
\hline Low & $256(35.6)$ & $196(22.9)$ & 1.00 & & & \\
\hline Normal $^{\S}$ & $466(64.4)$ & $661(77.1)$ & 1.867 & 1.498 & 2.327 & 0.001 \\
\hline \multicolumn{7}{|l|}{ Hepatitis } \\
\hline No & $612(84.4)$ & 781 (91.1) & 1.00 & & & \\
\hline Yes & $113(15.6)$ & $76(8.9)$ & 0.527 & 0.387 & 0.718 & 0.001 \\
\hline
\end{tabular}

${ }^{5} \geq 1.03$ (Male) and $\geq 1.30$ (Female). ARV: Antiretroviral. Adjusted for age, gender, ethnicity, hypertension, diabetes mellitus, CD4, HIV RNA Load, TG, LDL, HDL, FPG, alcohol consumption, smoking, liver disease, medication with agents including ARV (PI), b-blocker, anti-hyperglycemic. aOR Adjusted odds ratio.

attributed to treatment with PIs. The multiple mechanism of PIs in developing lipid and glucose abnormalities include reduction in catabolism and increase in production of very low density lipoprotein [51,52], impaired catabolism of fatty free acid [53], increased synthesis of triglyceride in liver [54], decreased expression of LDL receptors [55], interference with the intercellular process regulating glucose and lipid metabolism in insulin-response tissue [56]. Other relevant factors comprise of aging, abdominal obesity, diabetes, lifestyle, gender and ethnic differences, type and length of time on ARV regimen can result in various incidence/prevalence of lipid and glucose abnormalities.

Aging, race and gender differences are well-known irreversible risk factors for metabolic abnormalities. Elderly population has lipid and glucose metabolism changes due to liver cell dysfunctions [57]. The beneficial effect of endogenous estrogen women before menopause causes less lipid abnormalities [58]. Similar to this study Richter and colleagues [59] in a cohort study indicated that age, treatment with PI and male gender were risks for dyslipidemia. Some studies $[60,61]$ which were conducted in the United State also revealed that race/ethnicity was a highly significant predictor of plasma lipids. In a study the increased lipid level was significantly less associated with Malay and Chinese's race [62]. One of the possible explanations may be due to the lower prevalence of abdominal obesity among Malay and Chinese HIV patients [62]. In the present study alcohol consumption was associated with high level of TG. As the recognized dietary risk for 
Table 5 Risk factors for low high-density lipoprotein (HDL) level in 1582 HIV subjects (normal $=1128$, low HDL $=454$ ) on ARV medication

\begin{tabular}{|c|c|c|c|c|c|c|}
\hline \multirow[t]{2}{*}{ Characteristics } & \multirow{2}{*}{$\begin{array}{c}\text { Normal } \\
\text { Mean } \pm \text { S.D. } \\
\mathrm{N}(\%)\end{array}$} & \multirow{2}{*}{$\begin{array}{c}\text { Low HDL } \\
\text { Mean } \pm \text { S.D. } \\
\text { N (\%) }\end{array}$} & \multirow[t]{2}{*}{$\mathrm{aOR}$} & \multicolumn{2}{|c|}{ 95.0 C.I. for OR } & \multirow[t]{2}{*}{ p-value } \\
\hline & & & & Lower & Upper & \\
\hline \multicolumn{7}{|l|}{ Ethnicity } \\
\hline Malay & $105(23.3)$ & $285(25.4)$ & 1.00 & & & \\
\hline Indian & $62(13.7)$ & $96(8.6)$ & 0.570 & 0.386 & 0.842 & 0.009 \\
\hline Chinese & $284(27.8)$ & $739(72.2)$ & 1.753 & 1.187 & 2.589 & 0.005 \\
\hline \multicolumn{7}{|l|}{ ARV medication } \\
\hline Without PI & $382(86.2)$ & $998(90.1)$ & 1.00 & & & \\
\hline With PI & $61(13.8)$ & $110(9.9)$ & 1.449 & 1.037 & 2.024 & 0.029 \\
\hline \multicolumn{7}{|c|}{ Anti-hyperglycaemic Agents } \\
\hline Not taking & $57(12.6)$ & $91(8.1)$ & 1.00 & & & \\
\hline Taking & $397(87.4)$ & $1037(91.9)$ & 1.636 & 1.152 & 2.323 & 0.006 \\
\hline \multicolumn{7}{|c|}{ Viral Load (copies/mL) } \\
\hline$<20$ & $391(86.1)$ & $979(86.8)$ & 1.00 & & & \\
\hline$>20$ & $63(13.9)$ & $149(13.2)$ & 0.945 & 0.688 & 1.297 & 0.724 \\
\hline \multicolumn{7}{|c|}{ CD4 (cells/mm3) } \\
\hline$<200$ & $25(5.6)$ & $90(8.0)$ & 1.00 & & & \\
\hline$\geq 200$ & $419(94.4)$ & $1029(92.0)$ & 0.682 & 0.432 & 1.078 & 0.099 \\
\hline TG (mmol/l) & $2.08 \pm 1.37$ & $3.23 \pm 2.39$ & 0.693 & 0.646 & 0.745 & 0.001 \\
\hline$<5.17$ & $95(21.1)$ & $551(48.9)$ & 1.00 & & & \\
\hline$\geq 5.17$ & 356 (78.9) & $576(51.1)$ & 0.279 & 0.216 & 0.360 & 0.001 \\
\hline LDL (mmol/l) & $3.17 \pm 1.01$ & $2.64 \pm 1.05$ & 1.709 & 1.516 & 1.926 & 0.001 \\
\hline$<3.36$ & 349 (77.6) & $673(59.7)$ & 1.00 & & & \\
\hline$\geq 3.36$ & $101(22.4)$ & $454(40.3)$ & 2.331 & 1.813 & 2.997 & 0.001 \\
\hline \multicolumn{7}{|l|}{ TC (mmol/l) } \\
\hline$<5.17$ & $258(56.8)$ & $466(41.3)$ & 1.00 & & & \\
\hline$\geq 5.17$ & $196(43.2)$ & $661(58.7)$ & 1.867 & 1.498 & 2.327 & 0.001 \\
\hline \multicolumn{7}{|l|}{ Hepatitis } \\
\hline No & $383(84.4)$ & 1010 (89.5) & 1.00 & & & \\
\hline Yes & $71(15.6)$ & $118(10.5)$ & 0.630 & 0.459 & 0.865 & 0.004 \\
\hline
\end{tabular}

Adjusted for age, gender, ethnicity, hypertension, diabetes mellitus, CD4, HIV RNA Load, TC, TG, LDL, FPG, alcohol consumption, smoking, liver disease, medication with agents including ARV (PI), b-blocker, anti-hyperglycemic. aOR Adjusted odds ratio, ARV Antiretroviral.

high TG, alcohol is rich in calorie and disturb the liver function, the human organ which contributes in metabolize of the nutrients [63], thus it can be stored in body as fat and increase in blood as TG. It is important to stress that the data are derived from a male population mostly and that their relevance is mainly to be referred to male sex.

Interestingly the effect of some risk or protective factors on lipid and glucose can be explained by their indirect and intermediary role. In this study higher CD4 cell count was a risk factor for high TG while viral load level $<20\left(\right.$ copies $\left./ \mathrm{mm}^{3}\right)$ raised the risk of high TC. ARV medications boost immune system by increasing in CD4 cells and diminishing HIV vial load thus the effect of ARV medication on lipids was seen through the raised CD4 and decreased viral load [64]. We also found that treatment with anti-hyperglycemic agents was a significant associated risk for high TG and low HDL level. Since diabetic patients have more lipid abnormalities thus taking anti-hyperglycaemic medication can make a connection between diabetes and these lipid disorders indirectly [65]. Approximately $14 \%$ of our population had hepatitis as liver diseases who had lowered risk of lipid disorders and increased possibility of high FPG. On the hepatitis virus nature, some studies found that hepatitis viral replication during its metabolic processes can drop in lipid levels by interrupting cholesterol synthesis and using host lipids for replication, decreasing circulating lipids, and clearance of the virus results 
Table 6 Risk factors for increased fasting plasma glucose (FPG) level in 1540 HIV subjects (normal=952, increased FPG $=588$ ) on ARV medication

\begin{tabular}{|c|c|c|c|c|c|c|}
\hline \multirow[t]{2}{*}{ Characteristics } & \multirow{2}{*}{$\begin{array}{c}\text { Normal } \\
\text { Mean } \pm \text { S.D. } \\
\text { N (\%) }\end{array}$} & \multirow{2}{*}{$\begin{array}{c}\text { Hypertensive } \\
\text { Mean } \pm \text { S.D. } \\
\text { N (\%) }\end{array}$} & \multirow[t]{2}{*}{$\mathrm{aOR}$} & \multicolumn{2}{|c|}{ 95.0 C.I. for OR } & \multirow[t]{2}{*}{ p-value } \\
\hline & & & & Lower & Upper & \\
\hline \multicolumn{7}{|l|}{ Age (year) } \\
\hline Mean \pm SD & $42.79 \pm 9.68$ & $47.17 \pm 9.56$ & 1.048 & 1.036 & 1.059 & 0.001 \\
\hline \multicolumn{7}{|l|}{ Gender } \\
\hline Male & $751(78.9)$ & $494(84.0)$ & 1.00 & & & \\
\hline Female & $201(21.1)$ & $94(16.0)$ & 0.711 & 0.543 & 0.931 & 0.013 \\
\hline \multicolumn{7}{|l|}{ Hypertension } \\
\hline No & $754(82.6)$ & $398(70.8)$ & 1.00 & & & \\
\hline Yes & $159(17.4)$ & $164(29.2)$ & 1.954 & 1.522 & 2.508 & 0.001 \\
\hline \multicolumn{7}{|l|}{ b-Blocker } \\
\hline taking & $43(4.5)$ & $44(7.5)$ & 1.00 & & & \\
\hline Not Taking & $909(95.5)$ & $544(92.5)$ & 0.585 & 0.379 & 0.902 & 0.014 \\
\hline \multicolumn{7}{|c|}{ Anti-hyperglycaemic } \\
\hline Taking & $35(3.7)$ & $111(18.9)$ & 1.00 & & & \\
\hline Not taking & 917 (96.3) & $477(81.1)$ & 0.164 & 0.110 & 0.244 & 0.001 \\
\hline \multicolumn{7}{|c|}{ Diabetes mellitus } \\
\hline Yes & $96(10.1)$ & $149(25.3)$ & 1.00 & & & \\
\hline No & 856 (89.9) & $439(74.7)$ & 0.330 & 0.249 & 0.438 & 0.001 \\
\hline TG (mmol/l) & $2.25 \pm 1.74$ & $2.65 \pm 1.82$ & 1.130 & 1.066 & 1.199 & 0.001 \\
\hline$<5.17$ & $432(45.4)$ & 197 (33.6) & 1.00 & & & \\
\hline$\geq 5.17$ & $519(54.6)$ & $389(66.4)$ & 1.644 & 1.327 & 2.035 & 0.001 \\
\hline \multicolumn{7}{|l|}{ Hepatitis } \\
\hline No & $857(90.0)$ & $498(84.7)$ & 1.00 & & & \\
\hline Yes & $95(10.0)$ & $90(15.3)$ & 1.630 & 1.197 & 2.220 & 0.002 \\
\hline
\end{tabular}

Adjusted for age, gender, ethnicity, hypertension, diabetes mellitus, CD4, HIV RNA Load, TC, TG, LDL, HDL, alcohol consumption, smoking, liver disease, medication with agents including ARV (PI), b-blocker, anti-hyperglycemic. aOR Adjusted odds ratio.

in rebound increase of lipid levels [66,67]. Since liver is the main body organ for balancing plasma glucose, damage to the liver cell by hepatitis virus causes disturbed liver function and insulin production [68] thus it is expected the elevated FPG during hepatitis infection.

\section{Limitations of the study}

Comprehensive blood lipid and glucose evaluation were not assessed in this study just for those with current tests (year 2012). Also inability of assessment of anthropometrics including waist circumference, body mass index (BMI) and body fat percentage, the cross-sectional nature of the study, small number of female as well Indian subjects, and lack of ARVnaïve or HIV negative subjects as controls, absence of previous published studies in lipid abnormalities among Malaysian population with HIV/AIDS in order to comparison with the present study were considered as other limitations in this study.

\section{Conclusion}

Our study indicates a high proportion of HIV-infected patients receiving ARV medication met the criteria for dyslipidemia. Also treatment with protease inhibitorswas responsible and risk factor for high prevalence of lipid profile disorders. Therefore, it is crucial to evaluate and monitor these abnormalities before initiation and during Highly Active Antiretroviral therapy (HAART) to monitor any rising trends. It should be mentioned that study of metabolic abnormalities among ARV naive patients for assessment of independent effect of HIV itself on lipid and glucose is considerable due to the importance of the choice of type ARV agents in combination therapy as HAART.Moreover based on our results; assessment of long-term effects of ARVagents on lipid abnormalities is suggested. Also investigations and implementation programs on prevention and treatment of lipid abnormalities by the way of lifestyle and nutritional modification strategies are optional. 


\section{Abbreviations}

AIDS: Acquired immune deficiency syndrome; ART: Antiretroviral therapy; ARV: Antiretroviral; BMI: Body mass index; FPG: Fasting plasma glucose; HAART: Highly active antiretroviral therapy; HDL: High-density lipoprotein; HIV: Human immunodeficiency virus; LDL: Low-density lipoprotein; TC: Tota cholesterol; TG: Triglyceride.

\section{Competing interests}

The authors declared that they have no competing interest.

\section{Authors' contributions}

$\mathrm{HN}$ and RR designed research; HN conducted research and analysed data; KCLC supervised hospital settings. All authors read and approved the final manuscript.

\section{Acknowledgement}

We appreciate those contributed in this study including National University of Malaysia, Ministry of Health Malaysia, Sungai Buloh Hospital.

\section{Author details}

'Dietetics Program, School of Health Sciences, Faculty of Health Sciences, Universiti Kebangsaan Malaysia, Jalan Raja Muda Abdul Aziz 50300, Kuala Lumpur, Malaysia. ${ }^{2} J a b a t a n$ Perubatan Am, Hospital Sungai Buloh, Jalan Hospital Sungai Buloh, Selangor, Darul Ehsan 47000, Malaysia.

Received: 15 February 2013 Accepted: 31 July 2013

Published: 15 August 2013

\section{References}

1. The Joint United Nations Programme on HIV/AIDS: Global report: UNAIDS report on the global AIDS epidemic 2012. http://www.unaids.org/en/media/ unaids/contentassets/documents/epidemiology/2012/gr2012/ 20121120_unaids_global_report_2012_with_annexes_en.pdf. Accessed 10 November 2013.

2. Ministry of Health, Malaysia: Global AIDS Response 2012 Progress Report. Reporting Period: January 2010-december 2011. Putrajaya: Ministry of Health Malaysia; 2012

3. World Bank: Countryclassification. http://data.worldbank.org/country/malaysia. Accessed 10 November 2012

4. World Health Organization, The Joint United Nations Programme on HIV/AIDS and The United Nations Children's Fund: Global HIV/AIDS response: epidemic update and health sector progress towards universal access: progress report 2011. Geneva: World Health Organization; 2011.

5. World Health Organization: Antiretroviral therapy for HIV infection in adults and adolescents: recommendations for a public health approach. - $2010 \mathrm{rev}$. Geneva: World Health Organization; 2010.

6. Srivanich N, Ngarmukos C, Sungkanuparph S: Prevalence of and risk factors for pre-diabetes in HIV-1-infected patients in Bangkok, Thailand. JIAPAC 2010, 9(6):358-361.

7. Idiculla J, Ravindra'n GD, D'Souza J, Singh G, Furrugh S: Diabetes mellitus, insulin resistance, and metabolic syndrome in HIV-positive patients in South India. Int I Gen Med 2011, 4:73.

8. Sinxadi PZ, Mcllleron HM, Dave JA, Smith PJ, Levitt NS, Maartens G: Association of lopinavir concentrations and plasma lipid or glucose concentrations in HIV-infected South Africans. AIDS Res Ther 2012, 9(1):32

9. Wu PY, Hung CC, Liu WC, Hsieh CY, Sun HY, Lu CL, Chien KL: Metabolic syndrome among HIV-infected Taiwanese patients in the era of highly active antiretroviral therapy: prevalence and associated factors. J Antimicrob Chemother 2012, 67(4):1001-1009.

10. Butt AA, Fultz SL, Kwoh CK, Kelley D, Skanderson M, Justice AC: Risk of diabetes in HIV infected veterans pre-and post-HAART and the role of HCV coinfection. Hepatology 2004, 40(1):115-119.

11. Samaras K, Wand H, Law M, Emery S, Cooper D, Carr A: Prevalence of metabolic syndrome in HIV-infected patients receiving highly active antiretroviral therapy using international diabetes foundation and adult treatment panel III criteria associations with insulin resistance, disturbed body fat compartmentalization, elevated C-reactive protein, and hypoadiponectinemia. Diabetes Care 2007, 30(1):113-119.

12. De Wit S, Sabin CA, Weber R, Worm SW, Reiss P, Cazanave C, Phillips A: Incidence and risk factors for new-onset diabetes in HIV-infected patients the data collection on adverse events of anti-HIV drugs (D: A: D) study. Diabetes Care 2008, 31(6):1224-1229.

13. Capeau J, Bouteloup V, Katlama C, Bastard JP, Guiyedi V, Salmon-Ceron D, Chêne G: Ten-year diabetes incidence in 1046 HIV-infected patients started on a combination antiretroviral treatment. AIDS 2012, 26(3):303.

14. Tsiodras S, Mantzoros C, Hammer S, Samore M: Effects of protease inhibitors on hyperglycemia, hyperlipidemia, and lipodystrophy: a 5-year cohort study. Arch Intern Med 2000, 160(13):2050.

15. Hiransuthikul N, Hiransuthikul P, Kanasook Y: Lipid profiles of Thai adult HIV-infected patients receiving protease inhibitors. Southeast Asian J Trop Med Public Health 2007, 38(1):69-77.

16. Manuthu EM, Joshi MD, Lule GN, Karari E: Prevalence of dyslipidemia and dysglycaemia in HIV infected patients. E AFR MED J 2008, 85(1):10-17.

17. Yone EWP, Kengne AP, Ashuntantang G, Betyoumin AF, Ngogang J: Dyslipidaemia in HIV-1-infected patients receiving protease inhibitors after initial treatment with first-line-based non-nucleoside reverse transcriptase inhibitors: a cross-sectional study. BMJ open 2012, 2(4):1-5.

18. Friis-Moller N, Weber R, Reiss $P$, Thiébaut R, Kirk O, Monforte ADA, Lundgren JD: Cardiovascular disease risk factors in HIV patientsassociation with antiretroviral therapy:Results from the DAD study. Aids 2003, 17(8):1179-1193.

19. Tadewos A, Addis Z, Ambachew H, Banerjee S: Prevalence of dyslipidemia among HIV-infected patients using first-line highly active antiretroviral therapy in Southern Ethiopia: a cross-sectional comparative group study. AIDS Res Ther 2012, 9(1):31.

20. Fontas E, Van Leth F, Sabin CA, Friis-Møller N, Rickenbach M, DA Monforte A, Reiss P: Lipid profiles in HIV-infected patients receiving combination antiretroviral therapy: are different antiretroviral drugs associated with different lipid profiles? J Infect Dis 2004, 189(6):1056-1074.

21. Carr A, Samaras K, Chisholm DJ, Cooper DA: Pathogenesis of HIV-1-protease inhibitor-associated peripheral lipodystrophy, hyperlipidaemia, and insulin resistance. Lancet 1998, 351(9119):1881.

22. Carr A: HIV protease inhibitor-related lipodystrophy syndrome. Clin Infect Dis 2000, 30(Supplement 2):S135-S142.

23. Currier JS, Lundgren JD, Carr A, Klein D, Sabin CA, Sax PE, Smieja M: Epidemiological evidence for cardiovascular disease in HIV-infected patients and relationship to highly active antiretroviral therapy. Circulation 2008, 118(2):e29-e35.

24. Brown TT, Cole SR, Li X, Kingsley LA, Palella FJ, Riddler SA, Dobs AS: Antiretroviral therapy and the prevalence and incidence of diabetes mellitus in the multicenter AIDS cohort study. Arch Intern Med 2005, 165(10):1179.

25. Triant VA, Lee $H$, Hadigan C, Grinspoon SK: Increased acute myocardial infarction rates and cardiovascular risk factors among patients with human immunodeficiency virus disease. J Clin Endocrinol Metab 2007. 92(7):2506-2512

26. Maclnnes A, Lazzarin A, Di Perri G, Sierra-Madero JG, Aberg J, Heera J, Valdez H: Maraviroc can improve lipid profiles in dyslipidemic patients with HIV: results from the MERIT trial. HCT 2011, 12(1):24-36.

27. Friis-Moller N, Sabin CA, Weber R, D'ArminioMonforte A, El-Sadr WM, Reiss P, Lundgren JD: Combination antiretroviral therapy and the risk of myocardial infarction. New Engl J Med 2003, 349(21):1993-2003.

28. Biron A, Bobin-Dubigeon C, Volteau C, Piroth L, Perré P, Leport C, Biron C: Metabolic syndrome in French HIV-infected patients: prevalence and predictive factors after 3 years of antiretroviral therapy. AIDS Res Hum Retroviruses 2012, 28(12):1672-1678.

29. Mallon PW, Wand H, Law M, Miller J, Cooper DA, Carr A: Buffalo hump seen in HIV-associated lipodystrophy is associated with hyperinsulinemia but not dyslipidemia. JAIDS 2005, 38(2):156-162.

30. Law MG, Friis-Moller N, El-Sadr WM, Weber R, Reiss P, d'ArminioMonforte A Lundgren JD: The use of the Framingham equation to predict myocardia infarctions in HIV-infected patients: comparison with observed events in the D: A: D Study. HIV Med 2006, 7(4):218-230.

31. Achhra AC, Amin J, Hoy J, Tanuma J, Sirisanthana T, Nolan D, Giles M: Differences in lipid measurements by antiretroviral regimen exposure in cohorts from Asia and Australia. AIDS Res Ther 2012, 2012:1-9.

32. Estrada V, Bernardino Jl, Casado JL, Domingo P, Masiá M, Iribarren JA, Santos J: Prevalence of cardiovascular risk factors in Spanish HIV-infected patients on antiretroviral therapy.The CORONATOR Study [Abstract]. In Proceedings of the XIX International AIDS Conference 22-27 July 2012. Washingtone DC. http://pag. aids2012.org/Abstracts.aspx?AID=15438 
33. Kerr SJ, Duncombe C, Avihingsanon A, Ananworanich J, Boyd M, Sopa B, Ruxrungtham K: Dyslipidemia in an Asian population after treatment for two years with protease inhibitor-containing regimens. JIAPAC 2007, 6(1):36-46.

34. Domigos H, Cunha RVD, Paniago AMM, Martins DM, Elkhoury EB, Souza ASD: Metabolic effects associated to the highly active antiretroviral therapy (HAART) in AIDS patients. Braz J Infect Dis 2009, 13(2):130-136.

35. Armstrong C, Liu E, Grinspoon S, Okuma J, Spiegelman D, Guerino C, Hawkins C: Dyslipidemia in an HIV-positive, antiretroviral treatment-naïve population in Dar es Salaam, Tanzania. J Acquir Immune Defic Syndr 2011, 57(2):141.

36. Julius H, Basu D, Ricci E, Wing J, KusariBasu J, Pocaterra D, Bonfanti P: The burden of metabolic diseases amongst HIV positive patients on HAART attending the Johannesburg Hospital. Curr HIV Res 2012, 9(4):247-252.

37. Omech B, Sempa J, Castelnuovo B, Opio K, Otim M, Mayanja-Kizza H, Manabe YC: Prevalence of HIV-associated metabolic abnormalities among patients taking first-line antiretroviral therapy in Uganda. ISRN AIDS 2012, 2012:1-6.

38. Pharm SPMI: Prevalence of lipodystrophy in Thai-HIV infected patients. J Med Assoc Thai 2004, 87(6):605-611.

39. Homsanit M, Nelson KE, Sonjai A, Anekthananon T, Suwanagool S, Cofrancesco J Jr: Body shape and metabolic abnormalities in Thai HIVinfected patients. AIDS Res Hum Retroviruses 2007, 23(11):1314-1321.

40. Mankhatitham W, Luaengniyomkul A, Manosuthi W: Lipid profile changes in Thai HIV and tuberculosis co-infected patients receiving nonnucleoside reverse transcriptase inhibitors-based antiretroviral therapy. Journal of the Medical Association of Thailand. Chotmaihetthangphaet 2012, 95(2):163.

41. Pujari SN, Dravid A, Naik E, Bhagat S, Tash K, Nadler JP, Sinnott JT: Lipodystrophy and dyslipidemia among patients taking first-line, World Health Organization-recommended highly active antiretroviral therapy regimens in Western India. JAIDS 2005, 39(2):199-202.

42. Padmapriyadarsini C, Kumar SR, Terrin N, Narendran G, Menon PA, Ramachandran G, Swaminathan S: Dyslipidemia among HIV-infected patients with tuberculosis taking once-daily nonnucleoside reversetranscriptase inhibitor-based antiretroviral therapy in India. Clin Infect Dis 2011, 52(4):540-546.

43. Friedewald WT, Levy RI, Fredrickson DS: Estimation of the concentration of LDL-cholesterol. Clin Chem 1972, 18(6):499-515.

44. National Cholesterol Education Program (NCEP) Expert Panel on Detection, Evaluation, and Treatment of High Blood Cholesterol in Adults (Adult Treatment Panel III): Third report of the national cholesterol education program (NCEP) expert panel on detection, evaluation, and treatment of high blood cholesterol in adults (adult treatment panel III) final report. Circulation 2001, 106:3143-3421.

45. Grundy SM, Brewer HB, Cleeman Jl, Smith SC, Lenfant D, Conference Participants: Definition of metabolic syndrome: report of the national, heart, lung, and blood institute/american heart association conference on scientific issues related to definition. Circulation 2004, 109:433-438.

46. Grundy SM, Cleeman Jl, Daniels SR: Diagnosis and management of the metabolic syndrome: an American heart association/national heart, lung, and blood institute scientific statement. Circulation 2005, 112(17):2735-2752.

47. Centre for Disease Control and Prevention (CDC): Revised classification system for HIV infection and expanded surveillance case definition for AIDS among adolescents and adults. MMWR 1992, 41(RR-17):1-19.

48. Saag MS, Holodniy M, Kuritzkes DR: HIV viral load markers in clinical practice: recommendations of an International AIDS Society-USA Expert Panel. Nat Med 1996, 2:625-629.

49. SPSS 16.0: Statistical package for the social sciences 16.0. Chicago, Illinois: SPSS Inc; 2007

50. Chuapai Y, Kiertiburanakul S, Malathum K, Sungkanuparph S: Lipodystrophy and dyslipidemia in human immunodeficiency virus-infected Thai patients receiving antiretroviral therapy. J Med Assoc Thai 2007, 90(3):452-458.

51. Carpentier A, Patterson BW, Uffelman KD, Salit I, Lewis GF: Mechanism of highly active anti-retroviral therapy-induced hyperlipidemia in HIV-infected individuals. Atherosclerosis 2005, 178(1):165-172.

52. Shahmanesh M, Das S, Stolinski M, Shojaee-Moradie F, Jackson NC, Jefferson W, Umpleby AM: Antiretroviral treatment reduces very-low-density lipoprotein and intermediate-density lipoprotein apolipoprotein B fractional catabolic rate in human immunodeficiency virus-infected patients with mild dyslipidemia. Clin Endocrinol Metab 2005, 90(2):755-760.
53. Reeds DN, Yarasheski KE, Fontana L, Cade WT, Laciny E, DeMoss A, Klein S: Alterations in liver, muscle, and adipose tissue insulin sensitivity in men with HIV infection and dyslipidemia. Am J Physiol Endocrinol Metab 2006, 290(1):E47-E53.

54. Lenhard JM, Croom DK, Weiel JE, Winegar DA: HIV protease inhibitors stimulate hepatic triglyceride synthesis. Arterioscler Thromb Vasc Biol 2000, 20(12):2625-2629.

55. Petit JM, Duong M, Duvillard L, Florentin E, Portier H, Lizard G, Verges B: LDL-receptors expression in HIV-infected patients: relations to antiretroviral therapy, hormonal status, and presence of lipodystrophy. Am J Physiol EndocrinolMetab 2002, 32(5):354-359.

56. Hruz PW, Murata H, Mueckler M: Adverse metabolic consequences of HIV protease inhibitor therapy: the search for a central mechanism. Am J Physiol Endocrinol Metab 2001, 280(4):E549-E553.

57. Couteur L, David G, Cogger VC, Mccuskey RS, De Cabo RAFAEL, Smedsrod B, Fraser R: Age-related changes in the liver sinusoidal endothelium. Ann N Y Acad Sci 2007, 1114(1):79-87.

58. Habib SS, Aslam M, Hameed W: Gender differences in Lipids and Lipoprotein (A) profiles in healthy individuals and patients with type 2 Diabetes Mellitus. Pak J Physiol 2005, 1:1-2.

59. Richter A, Pladevall M, Manjunath R, Lafata JE, Xi H, Simpkins J, Irish W: Patient characteristics and costs associated with dyslipidaemia and related conditions in HIV-infected patients: a retrospective cohort study. HIV Med 2005, 6(2):79-90.

60. Foulkes AS, Woh DA, Frank I, Puleo E, Restine S, Megan LW: Associations among race/ethnicity, ApoC-III genotypes, and lipids in HIV-1-infected individuals on antiretroviral therapy. PLoS Med 2006, 3(3):0337-0347.

61. Gibert CL, Shlay JC, Sharma S, Bartsch G, Peng G, Grunfeld C: Racial differences in changes of metabolic parameters and body composition in antiretroviral therapy-naive persons initiating antiretroviral therapy. JAIDS 2009, 50(1):44-53.

62. Hejazi N, Lee MHS, Lin KG, Choong CLK: Factors associated with abdominal obesity among HIV-infected adults on antiretroviral therapy in Malaysia. GJHS 2010, 2(2):20.

63. Clerc O, Nanchen D, Cornuz J, Marques-Vidal P, Gmel G, Daeppen JB, Rodondi N: Alcohol drinking, the metabolic syndrome and diabetes in a population with high mean alcohol consumption. Diabetic Med 2010, 27(11):1241-1249.

64. Domingo P, Suarez-Lozano I, Teir R, Lozano F, Terrón A, Viciana P, Garrido M: Dyslipidemia and cardiovascular disease risk factor management in HIV-1-infected subjects treated with HAART in the Spanish VACH cohort. Open AIDS J 2008, 2:26

65. Scott R, O'Brien R, Fulcher G, Pardy C, D'Emden M, Tse D, Keech A: Effects of fenofibrate treatment On cardiovascular disease risk in 9,795 individuals with type 2 diabetes and various components of the metabolic syndrome the fenofibrate intervention and event lowering in diabetes (FIELD) study. Diabetes Care 2009, 32(3):493-498.

66. Perlemuter G, Sabile A, Letteron P, Vona G, Topilco A, Chrétien Y, Bréchot C: Hepatitis $C$ virus core protein inhibits microsomal triglyceride transfer protein activity and very low density lipoprotein secretion: a model of viral-related steatosis. The FASEB J 2002, 16(2):185-194.

67. Marzouk D, Sass J, Bakr I, El Hosseiny M, Abdel-Hamid M, Rekacewicz C, Fontanet A: Metabolic and cardiovascular risk profiles and hepatitis $C$ virus infection in rural Egypt. Gut 2007, 56(8):1105-1110.

68. Shintani Y, Fujie H, Miyoshi H, Tsutsumi T, Tsukamoto K, Kimura S, Koike K: Hepatitis $C$ virus infection and diabetes: direct involvement of the virus in the development of insulin resistance. Gastroenterology 2004, 126(3):840-848.

\section{doi:10.1186/1471-2458-13-758}

Cite this article as: Hejazi et al:: Metabolic abnormalities in adult HIV infected population on antiretroviral medication in Malaysia: a crosssectional survey. BMC Public Health 2013 13:758. 